\title{
Genomic characterization provides new insight into Salmonella phage diversity
}

\author{
Andrea I Moreno Switt ${ }^{1}$, Renato H Orsi ${ }^{1}$, Henk C den Bakker ${ }^{1}$, Kitiya Vongkamjan', Craig Altier ${ }^{2}$ \\ and Martin Wiedmann ${ }^{1 *}$
}

\begin{abstract}
Background: Salmonella is a widely distributed foodborne pathogen that causes tens of millions of salmonellosis cases globally every year. While the genomic diversity of Salmonella is increasingly well studied, our knowledge of Salmonella phage genomic diversity is still rather limited, despite the contributions of both lysogenic and lytic phages to Salmonella virulence, diversity and ecology (e.g., through horizontal gene transfer and Salmonella lysis). To gain a better understanding of phage diversity in a specific ecological niche, we sequenced 22 Salmonella phages isolated from a number of dairy farms from New York State (United States) and analyzed them using a comparative genomics approach.

Results: Classification of the 22 phages according to the presence/absence of orthologous genes allowed for classification into 8 well supported clusters. In addition to two phage clusters that represent novel virulent Salmonella phages, we also identified four phage clusters that each contained previously characterized phages from multiple continents. Our analyses also identified two clusters of phages that carry putative virulence (e.g., adhesins) and antimicrobial resistance (tellurite and bicyclomycin) genes as well as virulent and temperate transducing phages. Insights into phage evolution from our analyses include (i) identification of DNA metabolism genes that may facilitate nucleotide synthesis in phages with a G+C \% distinct from Salmonella, and (ii) evidence of Salmonella phage tailspike and fiber diversity due to both single nucleotide polymorphisms and major re-arrangements, which may affect the host specificity of Salmonella phages.

Conclusions: Genomics-based characterization of 22 Salmonella phages isolated from dairy farms allowed for identification of a number of novel Salmonella phages. While the comparative genomics analyses of these phages provide a number of new insights in the evolution and diversity of Salmonella phages, they only represent a first glimpse into the diversity of Salmonella phages that is likely to be discovered when phages from different environments are characterized.
\end{abstract}

Keywords: Salmonella phage, Phage genomics

\section{Background}

Salmonella is an important and globally distributed foodborne pathogen, which causes an estimated 93 million gastroenteritis cases, and 150,000 deaths annually, among the global human population [1]. In the United States, Salmonella causes an estimated 1 million annual human cases and is the leading reported cause of death and hospitalization related to foodborne disease [2]. This pathogen is principally acquired by the consumption of

\footnotetext{
* Correspondence: mw16@cornell.edu

'Department of Food Science, Cornell University, Ithaca 14853, New York Full list of author information is available at the end of the article
}

contaminated food, although contact with infected animals and human to human transmission are also known transmission routes of Salmonella [3]. Dairy cattle and dairy products are important sources of Salmonella; a number of serovars ranked in the top 10 Salmonella serovars among human cases in the U.S. (e.g., Newport, Typhimurium) are commonly isolated from dairy cattle [4-6]. Whereas several studies have reported the prevalence and distribution of Salmonella on dairy farms, there is limited data on Salmonella phage distribution in this environment. Recently, our group reported a high prevalence and diversity of Salmonella phages on dairy farms in rural areas of New York State, and also identified

\section{Biomed Central}


closely related phages on farms hundreds of miles apart [7]. We thus elected to use phage isolates from dairy farm environments as a model to explore the genomic diversity of Salmonella phages associated with a specific environment.

Bacteriophages are the most abundant biological entities on this planet, show a high degree of host specificity, and phages are present in all the environments where a suitable host is found [8]. Phage populations have been estimated to be highly dynamic, for example, it has been estimated that approx. $10^{23}$ phage infections per second occur globally in marine environments [9]. Consequently, phages play pivotal roles in bacterial evolution, from killing bacteria to being agents of horizontal gene transfer $[8,10,11]$. Several Salmonella phages and prophages have been reported (e.g., Fels-1, Gifsy-2, P22, FelixO1), and selected phages have been fully sequenced [8,12-16]. Currently, there are genome sequences available for Salmonella phages from different regions of the world (e.g., U.S., U.K., Canada, and South Korea), isolated from diverse animal production facilities (e.g., swine and poultry), and with different host specificity (e.g., able to lyse either Salmonella serovars Typhi, Typhimurium, or Enteritidis). Regardless of the previously available phage genome sequences, the diversity of Salmonella phages is still severely undersampled and our knowledge of the genomic diversity of Salmonella phages associated with different environments is very limited.

\section{Results and discussion}

The fact that Salmonella is well recognized as a diverse species, with $>2,600$ serovars, various patterns of transmission, and host specificity, has led to considerable recent efforts [17-19] to characterize the Salmonella pangenome and to probe the diversity of this pathogen at the genomic level. By comparison, our knowledge of Salmonella phage genomic diversity has remained rather limited; in big part due to under-sampling. We thus selected 22 Salmonella phages, with a range of different phenotypic and genotypic characteristics [7], from nine dairy farms for genome sequencing to characterize the Salmonella phage diversity in environments (dairy farm) and hosts (cattle) where Salmonella is commonly found. Major findings of this study include (i) identification, by cluster analysis, of two Salmonella phage orthoclusters that have not been previously reported, (ii) identification of four phage clusters containing phages previously identified in various locations worldwide, (iii) identification of phages carrying putative antimicrobial resistance and virulence genes, (iv) identification of DNA metabolism genes in Salmonella phages with $\mathrm{G}+\mathrm{C}$ contents different from Salmonella, and (v) evidence of Salmonella phage tailspike and fiber diversity due to both single nucleotide polymorphisms and major re-arrangements.

\section{Identification, by cluster analysis, of two Salmonella phage orthoclusters that have not been previously reported}

The 22 newly sequenced phage genomes ranged in size from 30 to $158 \mathrm{~kb}$ with $\mathrm{G}+\mathrm{C}$ contents ranging from 39 to $56 \%$ (Table 1); previously sequenced Salmonella phages ranged in genome size from 33 to $240 \mathrm{~kb}$ and showed $\mathrm{G}+\mathrm{C}$ contents between 39 to $53 \%[12,14,20,21]$ (see Additional file 1 for details). Annotation of the genomes sequenced here identified between 45 and 264 genes in a given phage, which is consistent with reports on previously sequenced Salmonella phages $[8,20]$. A total of 8 of the 22 phages sequenced here contained known lysogeny modules, suggesting a temperate life cycle (Table 1). To initially characterize the diversity of these phages, we clustered phage genomes based on the presence/absence of families of orthologous genes, similar to a method previously used to cluster Mycobacteriophage genomes [22-24]. We refer to this approach as an orthoclustering analysis, and will refer to the resulting clusters of phages as orthoclusters. A total of 65 phage genomes were included in this analysis, including the genomes for (i) 22 phages characterized here, (ii) 23 Salmonella phages that have previously been reported, and (iii) 20 phages that represent type species of known phage genera infecting Enterobacteriaceae [25] (Additional file 1). This analysis revealed eight distinct and well supported (100\% bootstrap support) clusters of phage genomes; 21 of the 22 new phage genomes reported here grouped into one of these eight well supported clusters (Figure 1A). Overall, the eight clusters identified here contained phages with high levels of overall genome homology. For example, the seven phages in cluster 1 shared 62/73 orthologous genes with amino acid (aa) identities for these genes ranging from 72 to $100 \%$ (93\% average aa identity across all orthologous genes). The five phages in cluster 4 shared 98/133 orthologous genes with aa identities for these genes ranging from 68 to $100 \%$ (91\% average aa identity across all orthologous genes). The genome wide nucleotide identity of phages sequenced here was also compared using a dot plot (Figure 1B), which supported the cluster relationships that were derived based on the orthocluster analysis. For example, phage FSL SP-101 falls in a separate branch from the other FSL phages in cluster 5 (FSL SP-031, FSL SP-038 and FSL SP-049; Figure 1A), and the dot plot analysis shows that FSL SP-101 displays low sequence similarity (as demonstrated by the interrupted line on the diagonal in the comparisons) when compared to the other phages in cluster 5 (Figure 1B), This result is in agreement with the considerable nucleotide divergence observed between FSL SP-101 and the other FSL phages in this cluster (e.g., FSL SP-101 and FSL SP-031 showed only 53.8\% nucleotide identity over the whole genome). 
Table 1 Characteristics of newly sequenced phages

\begin{tabular}{|c|c|c|c|c|c|c|c|c|c|}
\hline Phage & Farm & $\begin{array}{c}\text { Salmonella } \\
\text { serovar host }\end{array}$ & $\begin{array}{l}\text { Genome } \\
\text { size (kb) }\end{array}$ & GC \% & $\begin{array}{l}\text { Phage } \\
\text { cluster }^{2}\end{array}$ & $\begin{array}{l}\text { Putative } \\
\text { family }^{3}\end{array}$ & $\begin{array}{l}\text { Putative } \\
\text { life cycle }^{5}\end{array}$ & $\begin{array}{c}\text { Related } \\
\text { Salmonella phage } \\
\end{array}$ & $\begin{array}{c}\text { GenBank } \\
\text { accession no. }\end{array}$ \\
\hline FSL SP-030 & 8 & Dublin & 59 & 56.6 & 1 & Siphoviridae & temperate & SPN19 & KC139519 \\
\hline FSL SP-039 & 8 & Cerro & 59 & 56.6 & 1 & Siphoviridae & temperate & SPN19 & KC139514 \\
\hline FSL SP-088 & 10 & Typhimurium & 59 & 56.4 & 1 & Siphoviridae & temperate & SPN19 & KC139512 \\
\hline FSL SP-099 & 13 & Newport & 59 & 56.6 & 1 & Siphoviridae & temperate & SPN19 & KC139667- KC139680 \\
\hline FSL SP-019 & 3 & Newport & 59 & 56.4 & 1 & Siphoviridae & temperate & SPN19 & KC139571- KC139631 \\
\hline FSL SP-124 & 15 & Cerro & 59 & 56.5 & 1 & Siphoviridae & temperate & SPN19 & KC139515 \\
\hline FSL SP-029 & 8 & Dublin & 158 & 45.0 & 2 & Myoviridae & virulent & V01, SFP10 & KC139560- KC139570 \\
\hline FSL SP-063 & 9 & Dublin & 156 & 44.9 & 2 & Myoviridae & virulent & V01, SFP10 & KC139522- KC139525 \\
\hline FSL SP-058 & 3 & Dublin & 72 & 39.6 & 3 & Podoviridae & virulent & none & KC139517 \\
\hline FSL SP-076 & 9 & Dublin & 72 & 39.5 & 3 & Podoviridae ${ }^{4}$ & virulent & none & KC139520 \\
\hline FSL SP-010 & 2 & Mbandaka & 87 & 39.4 & 4 & Myoviridae & virulent & Felix 01 & KC139526- KC139542 \\
\hline FSL SP-012 & 1 & Mbandaka & 87 & 39.3 & 4 & Myoviridae & virulent & Felix 01 & KC139543- KC139556 \\
\hline FSL SP-107 & 13 & Mbandaka & 88 & 39.3 & 4 & Myoviridae & virulent & Felix 01 & KC139638- KC139648 \\
\hline FSL SP-031 & 8 & Cerro & 44 & 51.3 & 5 & Siphoviridae & virulent & SE2, SS3e & KC139518 \\
\hline FSL SP-038 & 6 & Cerro & 42 & 51.1 & 5 & Siphoviridae & virulent & SE2, SS3e & KC139652- KC139666 \\
\hline FSL SP-049 & 6 & Cerro & 43 & 50.9 & 5 & Siphoviridae & virulent & SE2, SS3e & KC139557- KC139559 \\
\hline FSL SP-101 & 13 & Dublin & 41 & 50.2 & 5 & Siphoviridae & virulent & SE2, SS3e & KC139511 \\
\hline FSL SP-062 ${ }^{1}$ & 9 & Newport & 56 & 42.8 & 6 & Siphoviridae $^{4}$ & virulent & none & KC139632- KC139637 \\
\hline FSL SP-069' & 9 & Newport & 56 & 42.8 & 6 & Siphoviridae & virulent & none & KC139649- KC139651 \\
\hline FSL SP-004 & 1 & Newport & 30 & 52.8 & 7 & Myoviridae $^{4}$ & temperate & P2, PSP3 & KC139521 \\
\hline FSL SP-126 & 15 & Kentucky & 51 & 42.9 & 8 & Siphoviridae & virulent & $\mathrm{T} 1$ & KC139513 \\
\hline FSL SP-016 & 2 & Anatum & 46 & 50.2 & - & Siphoviridae & temperate & Gifsy-2, Fels-1 & KC139516 \\
\hline
\end{tabular}

${ }^{1}$ FSL SP-062 and FSL SP-069 only differ in 4 single nucleotide polymorphisms; therefore, FSL SP-069 is a variant of FSL SP-062.

2 phage orthoclusters were determined based on presence/absence of orthologous genes.

3 putative families were determined in silico as described in Methods.

${ }^{4}$ families were also confirmed by transmission electron microscopy.

${ }^{5}$ presence of lysogeny module was used to identify the putative life cycle of a given phage isolate.

Phage FSL SP-016, a $46 \mathrm{~kb}$ phage, which contains a known lysogeny module (Table 1; Additional file 2), could not be assigned a specific cluster (Figure 1A). This phage clustered with the Salmonella phage Gifsy-2 and Fels-1 (although not supported by a significant bootstrap value [<50\%]) and shares 18/60 ORFs with Gifsy-2 [GenBank: NC_010393], with an average aa sequence identity of $87 \%$. In addition, two of the new phage genomes reported here (FSL SP-004 and FSL SP-126) grouped into clusters that only contained other phages isolated from the U.S. FSL SP-004 has a $30 \mathrm{~kb}$ genome that contains a known lysogeny module (Table 1, Additional file 2), indicating that this is a temperate phage. This phage groups into cluster 7 , along with $\mathrm{P} 2$, the type species of the genus P2likevirus. This genus also includes the Enterobacteria phage PSP3 [GenBank: NC_005340], which also infects Salmonella [26]; FSL SP-004 and PSP3 present an average aa identity of $92 \%$ among the 22 ORFs shared. FSL SP-126, a $51 \mathrm{~kb}$ phage (Table 1) that appears to be virulent (as no known lysogeny module was identified in the genome), clustered with Enterobacteria phage T1 [GenBank: NC_005833], which is the type species of Tunalikevirus. Among the 83 ORFs in FSL SP-126, 37 have an ortholog in T1 (the average aa identity for these 37 orthologs is approx. $90 \%$ ).

Five of the eight phage clusters identified here contain phages that were isolated from different farms (see Table 1). For example, cluster 1 contains 6 phages isolated from five farms (farms 3, 8, 10, 13, and 15), while cluster 4 contains 3 phages isolated from three farms (farms 1, 2, and 13). This finding is consistent with previous pulsed field gel electrophoresis (PFGE) and restriction fragment length polymorphism (RFLP) data [7], on these as well as additional phages, which also found evidence for isolation of similar phages on dairy farms hundreds of miles apart. Combined, these data suggest that these phages may either be genetically stable and endemic or easily dispersed among farms (e.g., through animal movement, traffic between farms, fomites that move between farms, etc.), consistent with the fact that Salmonella is commonly found on dairy farms $[4,27]$ and can be transmitted between farms through various means $[28,29]$. 


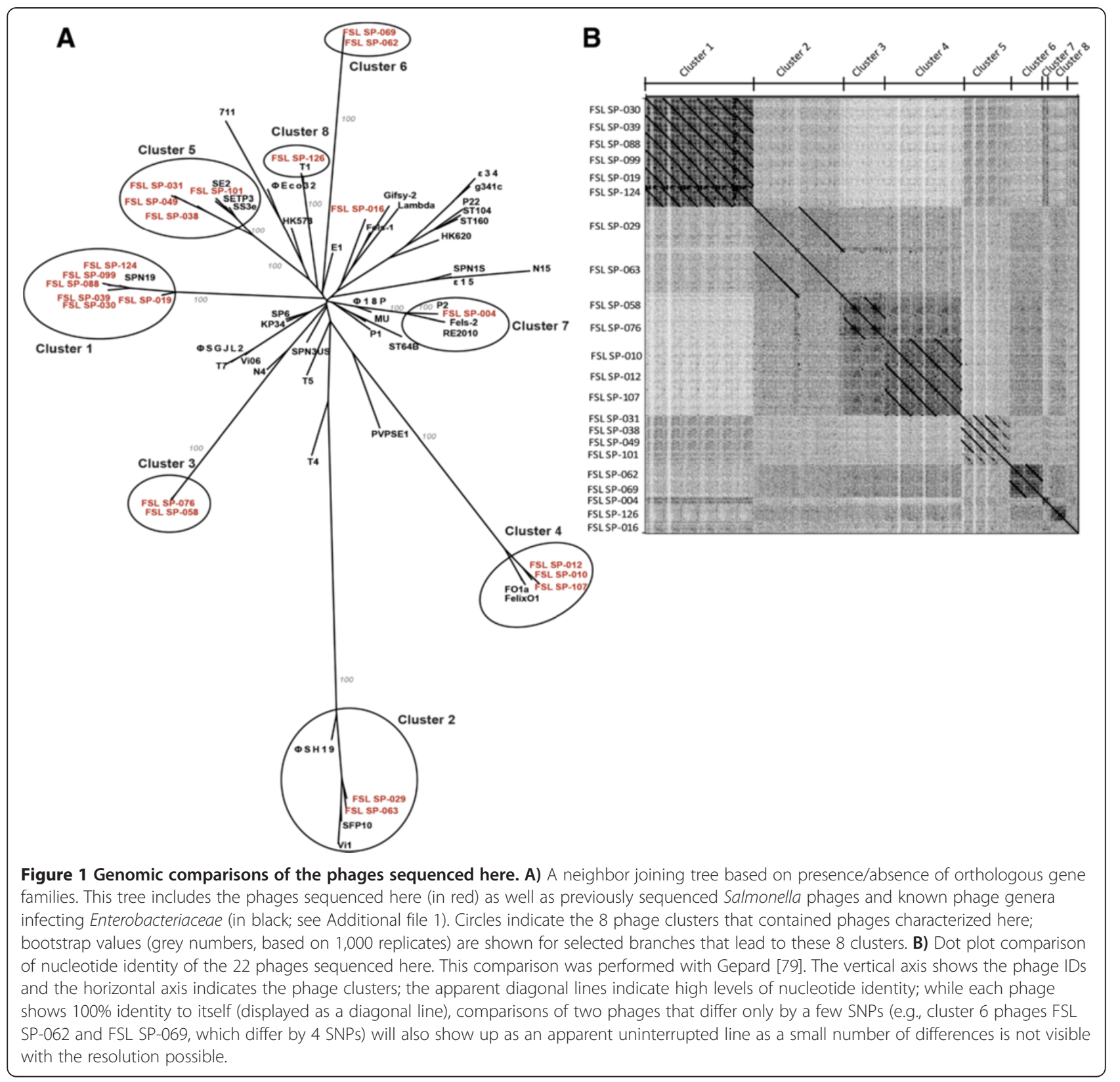

Two of the clusters identified here (clusters 3 and 6) only contained the genomes of Salmonella phages isolated here; these two clusters thus represent groups of Salmonella phages that have not been previously reported and their genomic characteristics are briefly described here. Cluster 3 includes two phages (FSL SP-058 and FSL SP-076; Table 1) that were obtained from 2 different farms. Ninety-seven ORFs were found in the phages of this cluster, functionally annotated ORFs include, for example, DNA polymerase, RNA polymerase, tailspikes, and terminase subunits (Additional file 2); aa identity among 88 orthologs found in both phages ranged from 65 to 100\% (89\% average aa identity). While these two phages do not contain a lysogeny module, suggesting that they are virulent, further phenotypic characterization of these phages is needed to confirm a virulent life cycle. Phages FSL SP-058 and FSL SP-076 share a conserved backbone, but show considerable variation in the two tailspike encoding genes (Figure 2A), which, as discussed below in detail, is often related with host range variability [30]. A BLASTP search of all cluster 3 ORFs against GenBank revealed no matching phages, suggesting that phages in cluster 3 appear to be novel virulent Salmonella phages.

Cluster 6 is the second cluster that includes only phages characterized here; the two phage isolates in this cluster (FSL SP-062 and FSL SP-069; Table 1) were obtained from 

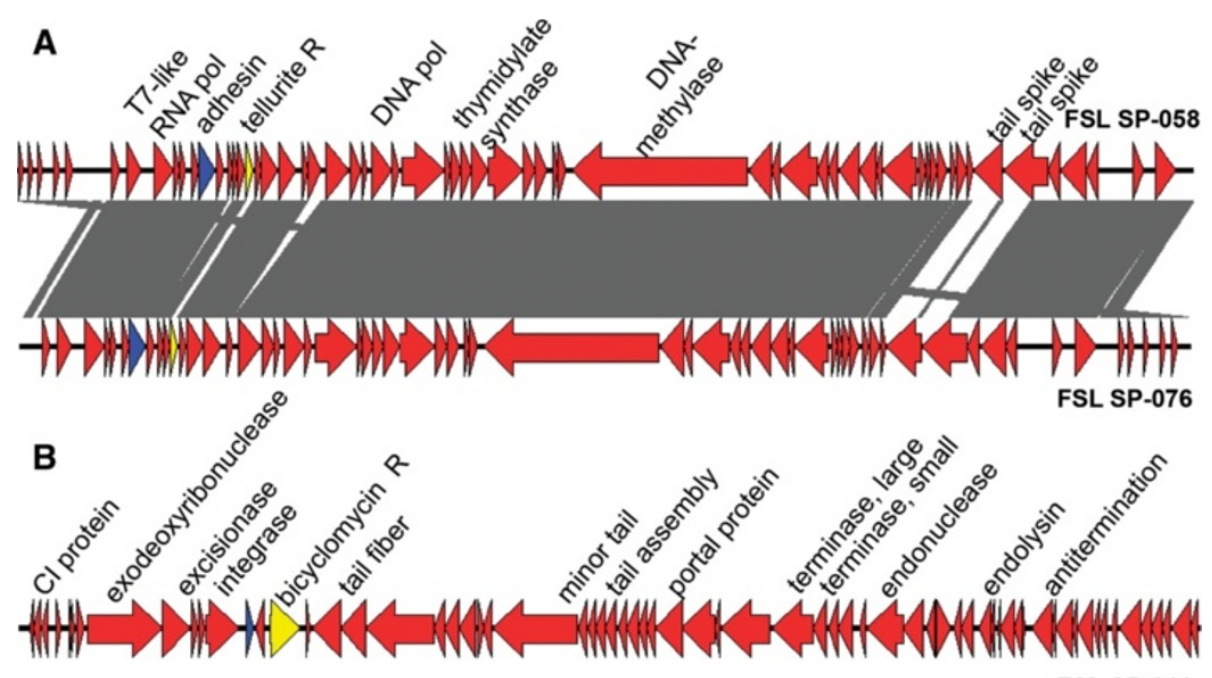

FSL SP-016

Figure 2 Linear representation of phages carrying antimicrobial resistance and virulence genes. (A) Comparison, using the BLAST algorithm, of phages in cluster 3 (FSL SP-058 and FSL SP-076), grey shaded regions are regions of homology between these two phages. (B) Linear representation of the temperate phage FSL SP-016. Red arrows indicate ORFs, blue arrows indicate putative virulence genes, and yellow arrows indicate putative antimicrobial resistance genes.

the same farm. We identified 102 ORFs in the phages of this cluster, 85 of them are hypothetical proteins and only 17 ORFs could be functionally annotated (Additional file 2 and Figure 3A). A lysogeny module is also absent in these two phages. Interestingly, only four single nucleotide polymorphisms (SNPs) differentiate phage isolates FSL SP-062 and FSL SP-069, with three of these SNPs located in the gene encoding the tail fiber; these two phage isolates thus should be considered variants rather than different phages (Table 1). Similar to cluster 3, a BLASTP search of all cluster 6 ORFs against GenBank revealed no matching phages, suggesting that the cluster 6 phages also represent novel Salmonella phages. Overall, we thus identified a number of novel Salmonella phages in this study, consistent with many reports that bacteriophages are extremely under-sampled and that with more environments and locations being sampled, more novel phages are likely to be identified [22,23,31].

\section{Identification of four phage clusters containing phages previously identified in various locations worldwide} In addition to two phage orthoclusters that only contained phages characterized here, we also identified four clusters (clusters 1, 2, 4, and 5) that each contains phages sequenced here as well as previously sequenced Salmonella phages obtained from other locations throughout the globe. Phage cluster 1 contains six very similar phages (isolated from 5 farms in the U.S.) as well as one phage that was isolated in South Korea (SPN19), which has not been assigned a genus to date [32]. These phages have a syntenic genome (Additional file 3), a size of approx. $59 \mathrm{~kb}$, and a $\mathrm{G}+\mathrm{C}$ content of 56.5\% (Table 1). Annotations identified genes encoding phage morphogenesis and replication proteins (Additional file 2). A lysis control module was identified in the 6 phage genomes sequenced here, but not in phage SPN19. Interestingly, phages FSL SP-030 and FSL SP-039, which cluster together based on the overall homology from the MAUVE alignment, were found to carry a different lysogeny control module (a Cro/C1 protein [33]) as compared to the other four phages, which encode a lysogeny control module that shows homology to the phage related helix-turn-helix XRE-family of transcriptional regulators [34]. Further analyses showed that the genomes of phages in cluster 1 resemble the genome of Enterobacter cancerogenes phage Enc34 [35] [GenBank: JQ340774], a phage of the Siphoviridae family that has not been assigned a genus to date, according to the International Committee on Taxonomy of Viruses 2012 [25]; this phage was hence not included in the cluster analysis shown in Figure 1. A Enterobacter phage Enc34 shows synteny with cluster 1 phages. While there are 11 hypothetical proteins present in phage Enc34, but not in the cluster 1 phages, 40 orthologs were found in both Enc34 and the cluster 1 phage FSL SP-088 (Additional file 4 ); the average aa identity among these shared ORFs is approx. $75 \%$. These data indicate that phages similar to cluster 1 have previously been isolated from Enterobacteriaceae hosts other than Salmonella.

Cluster 2 contains five Salmonella phages (Figure 1A), two sequenced in this study (obtained from 2 different farms) as well as three previously reported Salmonella phages (i.e., Vi1, ФSH19, SFP10, isolated from Canada, U.K., and South Korea, respectively) (Figure 1A). In addition, one previously reported Shigella phage (ФSboM-AG3, isolated 

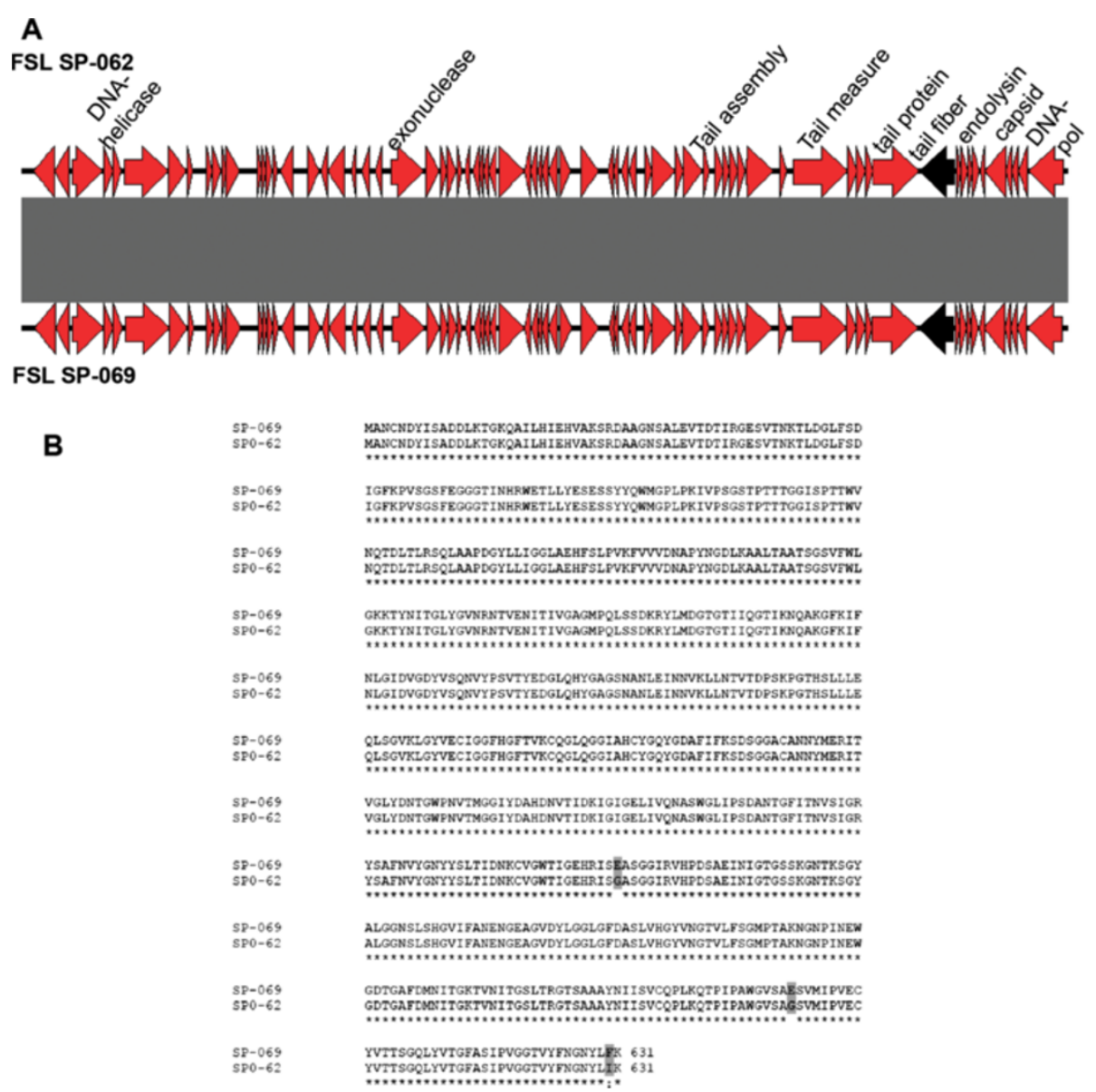

Figure 3 Linear representation of novel phages of cluster 6. (A) Comparison of FSL SP-062 and FSL SP-069 using the BLAST algorithm, red arrows indicate ORFs, black arrows indicate the tail fiber, and grey shaded regions are regions of homology between these two phages. (B) Alignment of the tail fibers amino acid sequences, the three shaded amino acids indicate substitutions.

from Canada) showed homology with the phages in this cluster (Figure 4A) [36]; a recent publication proposed these phages as "Viunalikevirus" [37], a new phage genus in the family Myoviridae. Phages belonging to cluster 2 possess large (approx. $158 \mathrm{~kb}$ ) genomes (Table 1); in the two phages sequenced here (i.e., FSL SP-029 and FSL SP-063), we identified 204 ORFs, 81 of them were functionally annotated (Additional file 2). While ORFs involved in phage morphogenesis, replication and DNA metabolism were found to be conserved among these six phages, genes involved in host specificity (i.e., tail fibers and tailspikes) showed considerable diversity (Figure 5), consistent with previous reports that these genes often show diversity due to recombination and therefore considerable mosaicism $[12,36,38,39]$. Despite their isolation from three continents, phages in cluster 2 present an overall genome synteny as well as high level conservation (aa identity of 65 to $100 \%$ across 115 homologs found in all 6 genomes; average aa identity of $89 \%$ ) (see Figure 4A). Interestingly, this conservation appears to be stable in both time and space;
Salmonella phage Vi1, which is the type species of this genus was isolated in Canada in the 1930's [38].

Cluster 4 includes three Salmonella phages sequenced in this study (i.e., FSL SP-010, FSL SP-012, and FSL SP-107, each isolated from a different farm), one Salmonella phage isolated in Switzerland (FO1a [GenBank: JF461087]), and one well characterized Salmonella phage isolated in the U.K. in 1943 (i.e., FelixO1) [40]. The five phages in this cluster have an approximate genome size of $87 \mathrm{~kb}$ (Table 1); aa identity among 98 shared orthologs ranged from 68 to $100 \%$ (average aa identity of approx. 91\%). FelixO1 is the type species of the genus Felixounalikevirus, this phage genus also includes Salmonella phage SPT-1, isolated in the U.S., E. coli phage wV8, isolated in the U.S., E. coli phage EC6, isolated in South Korea, and Erwinia phage ФEa21-4, isolated in Canada [21,40,41] (Figure 4B). We thus not only identified three new members of the genus Felixounalikevirus, but also provided further evidence that Felixounalikeviruses are widely distributed. Whole genome alignments, showed that phage FO1a is the closest to 

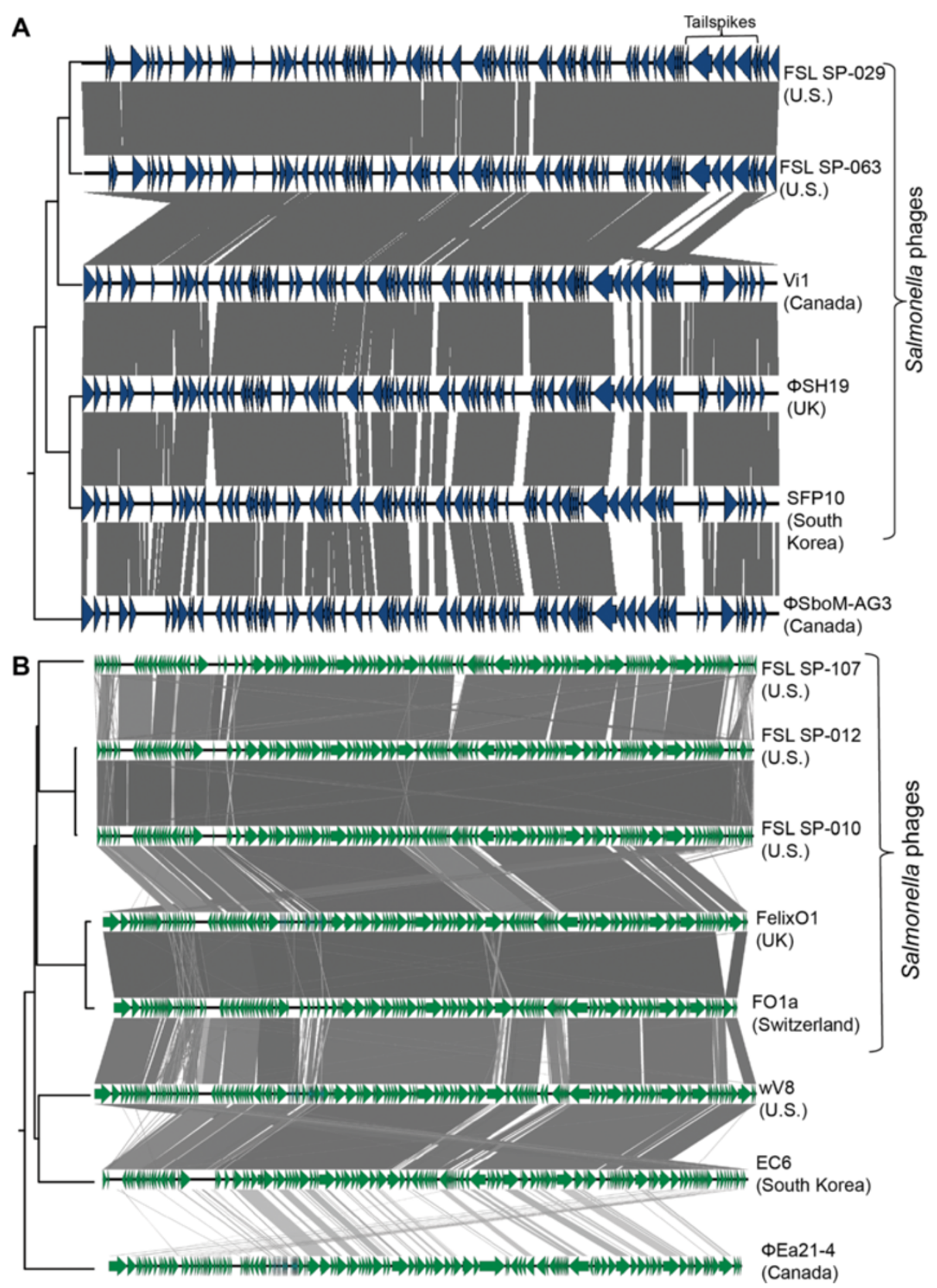

Figure 4 Comparison, using the BLAST algorithm, of phages in clusters 2 and 4. (A) Comparison of phages in cluster 2, representing two phages sequenced in this study (FSL SP-029 and FSL SP-063) and four phages previously reported in Canada, U.K., and South Korea, blue arrows indicate ORFs. (B) Comparison of phages in cluster 4, representing three phages sequenced in this study (FSL SP-010, FSL SP-012, and FSL SP-107) and five phages previously reported in Canada, U.S., South Korea, and U.K. Phages and country of origin are indicated on the right, green arrows indicate ORFs, and grey shaded regions are regions of homology. On the left side is the tree generated with the Mauve algorithm indicating the overall homology of the phages.

FelixO1, followed by the three Salmonella phages sequenced in this study (Figure 4B); the most distinct phage genome among the Felixounalikeviruses is Erwinia phage ФEa21-4, which only shares some ORFs with these phages, but lacks ORFs conserved among the rest of the phages in this genus (see Figure 4B). The major difference between FelixO1 and phages sequenced here were found to be insertions and deletions of homing endonucleases, which are mobile elements that have been reported in some phages [42]. Interestingly, genes encoding homing endonucleases are often inserted between genes or inserted into genes that appear conserved among these closely related phages. While high numbers of genes encoding homing endonucleases were reported for FelixO1 [40], we here 


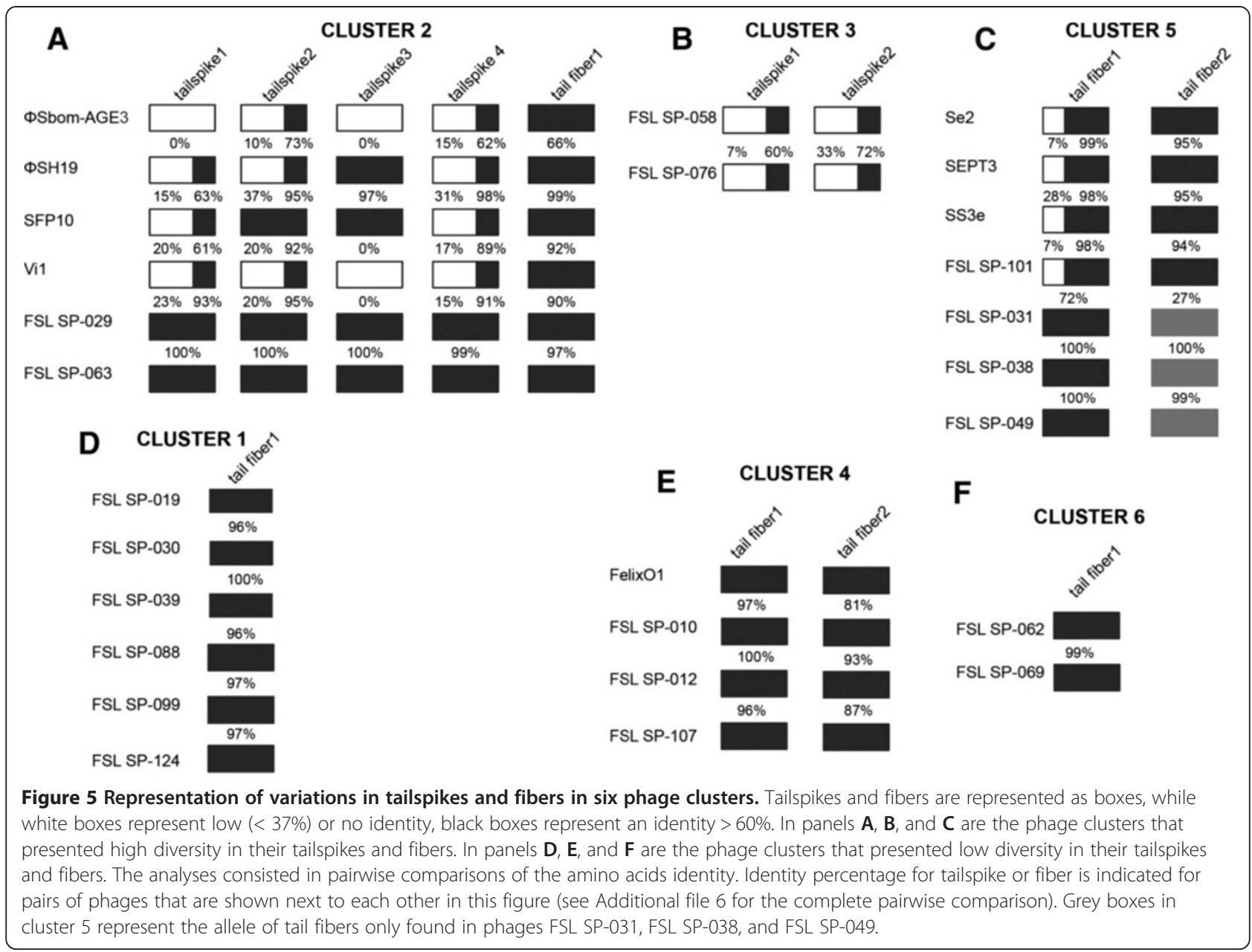

provided evidence that the presence of homing endonucleases appears to be common for Felixounalikeviruses.

Cluster 5 includes seven Salmonella phages, four phages sequenced in this study (i.e., FSL SP-031, FSL SP-038, FSL SP-049, and FSL SP-101, isolated from three different farms), two isolated in South Korea (i.e., SE2 and SS3e), and one isolated in the U.K. (i.e., SETP3) [43] (Figure 1A and Additional file 5). Phages in this cluster have a genome size of approx. $43 \mathrm{~kb}$ (Table 1); 67 ORFs were predicted among these phage genomes, including 43 annotated as hypothetical proteins and 24 that were functionally annotated (Additional file 2). Cluster 5 represents two groups of phages (see Figure 1A); group 1 represents three of the phages sequenced here (FSL SP-031, FSL SP-038, and FSL SP-049); these three phages are highly conserved (78 to $100 \%$ aa identity among 64 shared orthologs). The other group (group 2) contains three previously sequenced phages and one of the phages sequenced here (SETP3, SS3e, SE2, and FSL SP-101) (Additional file 5). Group 1 phages FSL SP-031 and FSL SP-038 have 96\% aa identity among 67 shared orthologs; the major differences between these two phages and FSL SP-049 are insertions and deletions of six hypothetical proteins and one homing endonuclease. This suggests that phages in this cluster are also hosts for homing endonucleases [40,42]. Each of the 7 phages in cluster 5 encodes a DNA/RNA helicase, which is a protein described to be involved in DNA replication and recombination [44] and could thus facilitate genomic rearrangements in phages in this cluster (Additional file 5). Overall, each of the four phage clusters discussed above contains not only phages from multiple farms, but also phages from at least two continents. In addition, two of these clusters contain the genomes of Salmonella phages that were isolated $>50$ years ago. Our findings here of closely related phages that are globally distributed are consistent with other genomic studies [22,37,45]; for instance, closely related Pseudomonas phages (up to 87\% DNA identity) were isolated from different countries in Europe and the U.S. $[46,47]$. Similarly, closely related Mycobacteriophages were isolated from up to four different continents (i.e., North America, Europe, Asia and Africa) [48,49]. Interestingly, some of these phage groups contain closely related phages that appear to have adapted to different hosts, including hosts representing different bacterial 
genera. While a number of studies suggest rapid diversification of phages, e.g., through recombination [50,51], our data suggest that at least some phage groups are relatively stable at the genomic level and also suggest that phage dispersal could contribute to global spread of transmissible genomic elements, including those encoding resistance and putative virulence genes.

\section{Identification of phages carrying putative antimicrobial resistance and virulence genes}

Virulence genes have been reported in a number of phages, including E. coli shiga toxigenic phages [52], and prophages, including the Salmonella prophages Gifsy-1 and Gifsy-2 [53-56]. In this study we describe the presence of putative antimicrobial resistance and virulence genes in genomes of virulent and temperate Salmonella phages. In the genome of FSL SP-016 we identified (i) one putative antimicrobial resistance gene, a homologue of $b c r$, which encodes a protein linked to bicyclomycin resistance. $b c r$ has been reported in a number of different Gram-negative and positive bacteria. Bicyclomycin is an antimicrobial compound, which inhibits the transcription terminator factor Rho [57]. This antimicrobial is obtained from cultures of Streptomyces spp. and is effective against Gram-negative bacteria, including Salmonella [58]. Bicyclomycin is used as feed additive in livestock in some countries, but this use of bicyclomycin is not approved in the U.S. [58]. However, this antimicrobial could be synthesized naturally by Streptomyces spp., which are commonly found in soil [59]. As FSL SP-016 is a temperate phage, presence of phage-encoded bicyclomycin resistance could be a selective advantage for the phage as it could facilitate survival of the host; however, we have no evidence for horizontal gene transfer or lysogen expression that would lead to phenotypic bicyclomycin resistance; further experiments are necessary to determine if the presence of $b c r$ can lead to phenotypic resistance.

In addition, each of the two phages in cluster 3 was found to carry (i) a putative virulence gene (a $y a d A$ homologue; $54 \%$ aa identity to Enterobacter sp. yadA) and (ii) a homologue of $\operatorname{ter} B$ (67\% aa identity with E. coli terB), which is part of a tellurite resistance operon (terZABCDEF) that has been found in the chromosome and plasmids of different bacteria [60,61] (Figure 2A \& Table 2). Similarly, a TerZ homolog was reported in Cronobacter phage vB_CsaP_GAP52 (GenBank acc. No. NC_019402). yadA was first identified in enteropathogenic Yersinia species [62] where it was reported to encode an agglutinating adhesin (YadA, Yersinia adhesion), a virulence factor that has been reported to mediate Yersinia adherence to epithelial tissue [62]. In addition to the phages reported here, a yadA homologue has also been identified in Enterobacteria phage ФEco32, Synechococcus phage metaG-MbCM1, Prochlorococcus phage P-HM2, and Pectobacterium phage
My1; an alignment of $\Phi E c o 32$ and the two phages of cluster 3 showed that they share no other regions of homology. Importantly, the genes encoding YadA homologs in FSL SP-058 and FSL SP-076 do not seem to locate close to genes encoding tail-morphogenesis proteins; rather, these genes are located next to a gene encoding a hypothetical protein and a gene encoding a deoxyuridine $5^{\prime}$-triphosphate nucleotide hydrolase. As both phages in cluster 3 did not contain a known lysogeny module, indicating that they are virulent phages, and did not show evidence for transduction (Table 3), it remains to be determined though if yadA can be transferred to Salmonella (or potentially other hosts) and if so if it would contribute to virulence in a recipient. As the function of $\operatorname{ter} B$ remains unknown [60] and as only one gene from the terZABCDEF was present in the cluster 3 genomes, it is difficult to assess the functional importance of carrying this gene, even though tellurite is known to be toxic for bacteria because it generates reactive oxidative species (ROS), which can damage metabolic enzymes and cause lipid peroxidation [63]. Importantly, these two phages were isolated from two different farms, suggesting a certain selective pressure to maintain $\operatorname{ter} B$, and the agglutinating adhesin.

In addition to carrying virulence and resistance genes, phages can also contribute to the dispersal of genes through lysogenic conversion and horizontal gene transfer [53]. We thus performed transduction assays, which found that 4/11 tested phages were able to transfer the chloramphenicol resistance gene from the $S$. Typhimurium donor strains to the recipient strain (see methods for details) (Table 3). The transduction frequency (ratio of transductants to PFU) [64] ranged from $9.0 \times 10^{-7}$ to $2.4 \times 10^{-5}$ (Table 3), which falls in the range previously reported for Salmonella phages. For example, Salmonella phage P22 had previously been shown to transduce at a frequency of $10^{-7}$ to $10^{-4}[64,65]$. Variations in transduction frequencies with different multiplicity of infections (MOI) and between replicates (Table 3 ) are consistent with previous reports [65,66]; for example, P22 has shown up to 1,000-fold differences in transduction frequency [65]. Interestingly, transducing phages belonged to two clusters, one identified in this study as representing temperate phages (cluster 1) and one previously identified as representing virulent phages (cluster 2). While transduction in temperate phages is easier to identify in laboratory conditions than in virulent phages (as virulent phages often lyse most of the transductants), a number of virulent phages have been identified as transducing phages [66]. This indicates that even if genome sequences indicate a virulent life cycle (e.g., absence of a known lysogeny module) and absence of phage-borne virulence and resistance genes, in vitro transduction tests are needed before a phage can be considered as safe to use as biocontrol agent. 
Table 2 Antimicrobial resistance, virulence and DNA metabolism genes identified in phages sequenced in this study

\begin{tabular}{|c|c|c|}
\hline Function & Gene & Phages harboring this gene \\
\hline \multirow[t]{2}{*}{ Resistance } & Tellurite resistance, TerB & Cluster 3 \\
\hline & Bicyclomycin resistance protein & FSL SP-016 \\
\hline \multirow[t]{2}{*}{ Virulence } & Agglutinating adhesin & Cluster 3 \\
\hline & Virulence protein MsgA/putative damage inducible protein Dinl ${ }^{1}$ & FSL SP-016 \\
\hline \multirow[t]{16}{*}{ DNA metabolism } & Thymidylate synthase & Clusters 2, 3, 4 \\
\hline & Ribonucleotide reductase of class III, large subunit & Clusters 2, 3, 4 \\
\hline & Ribonucleotide reductase of class III, activating subunit & Clusters 2, 3, 4 \\
\hline & Glutaredoxin & Clusters 2, 4 \\
\hline & Deoxyuridine 5'-triphosphate nucleotidohydrolase & Clusters 3,6 \\
\hline & Dihydrofolate reductase & Cluster 4 \\
\hline & Ribose-phosphate pyrophosphokinase & Cluster 4 \\
\hline & Ribonucleotide reductase of class la, alpha subunit & Cluster 4 \\
\hline & Ribonucleotide reductase of class la, beta subunit & Cluster 4 \\
\hline & Exodeoxyribonuclease & Cluster 4 \\
\hline & Ribose phosphate pyrophosphokinase & Cluster 4 \\
\hline & Nicotinamide phosphoribosyltransferase & Cluster 4 \\
\hline & Deoxynucleotide monophosphate kinase & Cluster 4 \\
\hline & Ribonuclease HI & Cluster 2 \\
\hline & dCMP deaminase & Cluster 2 \\
\hline & Nucleoside 2-deoxyribosyltransferase & Cluster 1 \\
\hline
\end{tabular}

\footnotetext{
${ }^{1}$ This protein shows homology with both Dinl ((pfam06183 [1.35e-08]) and with MsgA, (97\% of aa identity with Gifsy-2 prophage MsgA). Experimental data will be needed to assign a functionally appropriate name to this gene.
}

\section{Identification of DNA metabolism genes on Salmonella phages with $\mathrm{G}+\mathrm{C}$ contents different from Salmonella}

In five phage clusters (cluster 1, 2, 3, 4 and 6) we identified a number of genes involved in DNA metabolism. All of these clusters represented phages with a $\mathrm{G}+\mathrm{C}$ content different from the Salmonella genome $(\mathrm{G}+\mathrm{C}$ content of approx. 50-52\%); phages in clusters 1, 2, 3, 4, and 6 showed average $\mathrm{G}+\mathrm{C}$ contents of $56 \%, 45 \%, 39 \%, 39 \%$, and $42 \%$, respectively (Table 1 ). However, in phages with a $\mathrm{G}+\mathrm{C}$ content like Salmonella (cluster 5, cluster 8, and FSL SP-016, G+C content of $51 \%, 52 \%, 50 \%$, respectively), no ORFs were annotated as DNA metabolism genes. The number of DNA metabolism genes ranged from 1 to 12 genes per phage. For example, all phages in cluster

Table 3 Transduction frequency of chloramphenicol resistance for sequenced Salmonella phages that infected S. Typhimurium donor and recipient

\begin{tabular}{|c|c|c|c|c|c|c|}
\hline \multirow[b]{2}{*}{ Salmonella Phage } & \multicolumn{3}{|c|}{ Transduction frequency ${ }^{\mathrm{a}}$ in Replicate 1} & \multicolumn{3}{|c|}{ Transduction frequency ${ }^{\mathrm{a}}$ in Replicate 2} \\
\hline & MOI 0.1 & MOI 1 & MOI 10 & MOI 0.1 & MOI 1 & MOI 10 \\
\hline FSL SP-019 & - & - & - & - & - & - \\
\hline FSL SP-030 & - & - & $2.0 \times 10^{-6}$ & - & - & - \\
\hline FSL SP-039 & - & - & - & - & - & - \\
\hline FSL SP-088 & - & $2.4 \times 10^{-5}$ & $4.5 \times 10^{-6}$ & - & - & - \\
\hline FSL SP-099 & - & - & - & - & - & - \\
\hline FSL SP-124 & - & - & - & - & - & - \\
\hline FSL SP-063 & - & $4.0 \times 10^{-6}$ & $7.0 \times 10^{-7}$ & $1.0 \times 10^{-5}$ & $4.0 \times 10^{-6}$ & $1.2 \times 10^{-6}$ \\
\hline FSL SP-029 & - & - & $9.0 \times 10^{-7}$ & $2.0 \times 10^{-5}$ & $7.0 \times 10^{-6}$ & $8.0 \times 10^{-7}$ \\
\hline FSL SP-058 & - & - & - & - & - & - \\
\hline FSL SP-076 & - & - & - & - & - & - \\
\hline FSL SP-101 & - & - & - & - & - & - \\
\hline
\end{tabular}

${ }^{\mathrm{a}}$ Transduction frequency was calculated as the number of transductants/PFU used for transduction; MOI multiplicity of infection. 
4 (i.e., Felixounalikeviruses) carry 12 genes annotated as playing a role in DNA metabolism (Table 2). The same DNA metabolism genes had previously been identified in phages FelixO1 and ФEa21-4 [40,41], suggesting that these genes are part of the genomic backbone of this phage genus. Phages in cluster 2 included six DNA metabolism genes (Table 2). These genes also appear to be part of the backbone of this phage cluster as supported by identification of these genes in closely related phages (e.g., Vi1, SFP10; see Figure 4A) that also grouped in this cluster [12,36,38,39]. DNA metabolism genes have previously been reported in a number of phages $[12,21,36,38,40,67]$; the best characterized phage carrying DNA metabolism genes is bacteriophage T4 $[67,68]$. In $\mathrm{T} 4$, proteins involved in DNA metabolism, replication, and repair function in a complex called T4 nucleotide precursor complex $[67,68]$, which converts cellular nucleotide precursors into deoxynucleotide triphosphates with a different $\mathrm{G}+\mathrm{C}$ ratio than the host [67]. As T4 has a $\mathrm{G}+\mathrm{C}$ content of $34.5 \%$, it uses the nucleotide precursor complex to adjust the host's nucleotide ratios (approx. 50\% $\mathrm{G}+\mathrm{C}$ ) to ratios needed for $\mathrm{T} 4$ replication [67]. Our data indicate that similar mechanisms may be at play to facilitate replication of Salmonella phages whose $\mathrm{G}+\mathrm{C}$ content differ from their host. Expression of DNA metabolism genes may thus be a common mechanism used to debottleneck DNA replication in some phages with a $\mathrm{G}+\mathrm{C}$ content different from their hosts.

\section{Evidence of Salmonella phage tailspike and fiber diversity due to both single nucleotide polymorphisms and major re-arrangements}

Tail fibers and tailspikes are appendages in the phage tail that facilitate the initial binding of the phage to the bacterial host; they hence represent primary receptor-binding proteins. Phages can have one or more tailspikes or fibers, or combinations of tailspikes and fibers [69]. These virion structures target proteins or polysaccharides in the host surface, and therefore, have a role in host specificity $[69,70]$; nucleotide polymorphisms, duplications and rearrangements in the tail fiber and tailspike encoding genes appear to be associated with changes in the host range [30]. As our initial analysis (see above) found evidence for (i) major re-arrangements in genes encoding tailspikes and fibers in some orthoclusters (clusters 2, 5, and 3) and (ii) small number of SNPs in the tail fibers of the three other orthoclusters (clusters 1, 4, and 6), we performed more detailed analyses to characterize the genetic diversity in genes encoding tailspikes and fibers.

In the six phages in orthocluster 2, the region that contains the four genes encoding tailspikes is by far the most variable region of the genome (Figure 5A \& Additional file 6); however, the tail fiber gene is conserved among all six phages (pairwise aa identities ranged from
66 to 97\%; Additional file 6). In addition, for two phages in this cluster (FSL SP-029 and FSL SP-063, isolated from different farms), overall aa identities in all tailspikes and the tail fiber were high (97-100\%; Figure 5A). Highly variable tailspikes typically presented a fairly conserved N-terminal, with low aa identities in the $\mathrm{C}$ terminal (10 to $37 \%$, except for phages FSL SP-029 and FSL SP-063) (Figure 5A). These findings are consistent with Hooton et al. [12], who previously analyzed the tailspikes in three phages of the Viunalikevirus genus (ФSH19, Vi1, ФSbom-AG3); conservation of the $\mathrm{N}$-terminal residues is also consistent with the fact that this region attaches to the baseplate, while the remainder of the protein projects from the surface and appears to be involved in initial binding to the host $[12,69]$. Although future experimental work is needed, a role of the C-terminal variable regions in phage host specificity is supported by the observation that one of the tailspikes of the Salmonella Typhi specific phage Vi1 has a conserved acetyl esterase domain that recognizes the Typhi capsule as receptor [38]. Phage SFP10, which was reported to infect both Salmonella and E. coli O157:H7, also carries tailspikes that presented similarity to tailspikes found in both E. coli and Salmonella phages [39], suggesting a possible role of these regions in host specificity.

Phages in orthocluster 3 (FSL SP-058 and FSL SP-076) encoded two tailspikes, these proteins also presented a conserved $\mathrm{N}$-terminal and a highly divergent $\mathrm{C}$-terminal (7 and 33\% aa identity) (Figure 5B); these two phages show considerable differences in host range (2 and 3 Salmonella host strains are only lysed by FSL SP-058 and FSL SP-076, respectively; see Additional file 7). While the two tail fibers in the orthocluster 5 phages typically show a higher level of conservation and a longer conserved $\mathrm{N}$-terminal region, the C-terminal region of one tail fiber (tail fiber 1 ) still shows considerable variability in four of these phages (Figure $5 \mathrm{C} \&$ Additional file 6). The second tail fiber gene in cluster 5 presented two distinct alleles; phages FSL SP-031, FSL SP-038 and FSL SP-049 share one conserved tail fiber ( 99 to $100 \%$ aa identity) as do phages Se2, SEPT3, SS3e, and FSL SP-101 (94 to 95\% aa identity), with only $27 \%$ average aa identity between these two tail fibers (see Additional file 6).

Phages in orthoclusters 1,4 , and 6 , on the other hand, showed low divergence in their tail fibers. For example, among the four phages in orthocluster 4, tail fiber 1 was highly conserved (96 to $100 \%$ aa identity, Figure 5E), while tail fiber 2 showed 81 to $93 \%$ aa identity among these phages (Figure 5E). Interestingly, these phages differ considerably in their host range; FelixO1 infected all 23 Salmonella strains tested (in a previous report [71] FelixO1 lysed 98.2\% of Salmonella isolates tested), while FSL SP-010, FSL SP-012, and FSL SP-107 present a narrower host range infecting 4,7 and 3 strains, respectively (see Additional file 7). Further experimental 
and comparative genomics studies of these phages may thus provide an opportunity to understand why some phages such as FelixO1 have an extremely wide host range. The two phage variants in cluster 6 showed $100 \%$ aa identity across the proteins they encode except in the tail fiber, which presented three amino acids changes (Figure 3B); two substitutions of glutamic acid to glycine and one of phenylalanine to isoleucine (Figure 3B). While these two phage variants were isolated from the same farm, FSL SP-062 infects $S$. Newport and S. Kentucky, but FSL SP-069 only infects $S$. Newport (Additional file 7). Experimental studies are necessary to validate the role of these mutations in the increase of FSL SP-062 host range. Further studies on the contributions of different diversification patterns on phage host specificity, facilitated by the comparative genomics studies reported here, will provide a possibility to advance towards a more rational approach to constructing both narrow and wide host range Salmonella phages, which may open new opportunities in phage based detection and biocontrol.

\section{Conclusions}

This study used a genomic approach to investigate the diversity of 22 newly sequenced Salmonella phages. A number of novel Salmonella phages were identified, representing phages different from currently known phage genera infecting Enterobacteriaceae. Comparative genomics analyses of these phages have provided a number of new insights in the evolution and diversity of Salmonella phages, such as identification of a possible wide-spread role of DNA metabolism genes in facilitating replication of phages with GC contents distinct from the GC content of Salmonella. Our data also elude to the possibility of distinct mechanisms, among different phage families, of tail fiber and tailspike diversification, which may be linked to evolution of host specificity. Clearly, our analyses reported here only provide a first glimpse into the diversity of Salmonella phages that is likely to be discovered when phages from different environments are characterized by full genome sequencing and metagenomic approaches.

\section{Methods}

\section{Phage isolates}

A total of 22 phages were selected for whole genome sequencing (Table 1). These phages were previously isolated and characterized, including for host range and genome size, by pulsed field gel electrophoresis [7]. According to these previously characterized features, these phages were selected to represent diversity within our collection, including phages with narrow and wide host range, phages that infect different Salmonella serovars, and phages isolated from different farms in New York State. Phage lysate preparation and DNA extraction was conducted as previously described $[7,72]$. Briefly, DNA extraction was performed with phenol/chloroform, followed by ethanol precipitation. DNA was dissolved in $50-100 \mu \mathrm{l}$ of TE buffer $(10 \mathrm{mM}$ Tris, 1 mM EDTA; pH 8.0) and quantified using $\mathrm{OD}_{260}$ values measured with a Nanodrop Spectrophotometer (NanoDrop products, Wilmington, DE).

\section{Sequencing and annotation workflow}

Phage genomes were sequenced with the Illumina Genome Analyzer IIx (Illumina Inc. San Diego, CA) at the Cornell University Life Sciences Core Laboratories Center. Fiftybase pair reads were assembled de novo using the Velvet algorithm [73]. For 11 phages, the genome was assembled into one single contig; in phages with multiple contigs a pseudogenome was prepared for comparison purposes. Briefly, contigs were ordered according to their alignment to a reference phage (phage that presented homology among the phages sequenced in this study or previously sequenced phages). Then, contigs were merged with a pseudomarker (nnn), which was added to identify the different contigs. Contigs were annotated using a combination of automatic annotations by RAST [74], and the NCBI Prokaryotic Genomes Automatic Annotation Pipeline (PGAAP) [75], followed by manual curation using RAST. Sequences are available in Additional file 8, and pseudogenomes are available at the Cornell Food Safety Laboratory Microbial Genome Data Wiki page (https:// confluence.cornell.edu/display/FOODSAFETY/Cornell+ Food+Safety+Laboratory+Microbial+Genome+Data) [76] . A summary of annotations for the phages sequenced here is available in Additional file 2.

\section{Clustering and comparative analysis}

To classify phages into clusters, an orthologous gene presence/absence matrix was created using OrthoMCL v.1.4 [77] with the default settings. This matrix with the presence and absence of predicted proteins was used to prepare a neighbor-joining tree using Splits Tree4 [78]. This cluster analysis included genomes of (i) 20 nonredundant phages that represent the type species of all known phage genera infecting the Enterobacteriaceae [25], (ii) 23 previously reported Salmonella phages (representing all Salmonella phages available in http://www.ebi.ac. uk/genomes/phage.html as of August, 2012), and (iii) 22 phages reported and sequenced here (Additional file 1). Genome-wide nucleotide comparisons were performed using Gepard 1.3 [79] and the concatenated genome sequences of the 22 phages sequenced here. To facilitate the display of the nucleotide similarities as a dot plot, phage genomes were ordered according to their placement in the previously identified orthoclusters.

Whole genome alignments were conducted with Mauve [80], and the guide tree was used to visualize overall phages 
similarity. Comparisons were conducted within clusters with Mauve and RAST. Nucleotide and amino acid sequence alignments, and pairwise comparisons were conducted in MegAlign (DNASTAR Inc, Madison, WI), with the ClustalW algorithm. Linear representations of the whole genome BLAST comparisons were produced with Easyfig [81].

For each phage, all predicted ORFs were searched against GenBank using the BLASTP algorithm [82]. Hits in this search were used to identify similar phage genomes already in GenBank; similarity with previous phages was also used to aid with classification of newly sequenced phages into putative phage families. Phages in which a known lysogeny module was annotated were classified as putative temperate phages, phages without a known lysogeny module were classified as putative virulent phages $[38,39]$.

\section{Bacteriophage mediated-transduction}

Transduction assays were conducted as described for Salmonella phage P22 [65]. These assays were conducted only on phages that infected $S$. Typhimurium donor and recipient strains (Table 3 ). For donor strain we used S. Typhimurium FSL R8-3980, which has a chromosomally inserted cat gene (chloramphenicol resistance); as recipient we used the wild type strain FSL R8-3981. A phage lysate was prepared using the donor strain; for this, $200 \mu \mathrm{l}$ of an overnight culture of the donor, a phage concentration of $5 \times 10^{6} \mathrm{PFU} / \mathrm{ml}$, and $1 \mathrm{ml}$ of LB broth (Bacto, Franklin Lakes, NJ) were mixed and incubated for $12-16 \mathrm{~h}$ at $37^{\circ} \mathrm{C}$. Phage lysate was recovered by adding 4-5 drops of chloroform, followed by centrifugation for $10 \mathrm{~min}$ at $10,000 \mathrm{rpm}$; the lysate was then titered. For transduction, this lysate was used to infect the recipient. Briefly, an overnight culture of the recipient was diluted 50fold into $\mathrm{LB}$ broth, and incubated at $37^{\circ} \mathrm{C}$ to $\log$ phase (1.5-3 h), phage was then added in three different multiplicity of infections (i.e., 0.1, 1, and 10), and lysate was incubated at $37^{\circ} \mathrm{C}$ for $30 \mathrm{~min}$. To avoid secondary infections $0.5 \mathrm{ml}$ of $20 \mathrm{mM}$ EGTA (Fisher scientific, Pittsburgh, PA) was added, and lysate was incubated for $1 \mathrm{~h}$ at $37^{\circ} \mathrm{C}$ with shaking. Samples were centrifuged, and pellet was suspended in $100 \mu \mathrm{l}$ of LB broth. All $100 \mu \mathrm{l}$ were then spread on plates prepared with $20 \mu \mathrm{g}$ of chloramphenicol (Sigma-Aldrich, St. Louis, MO) and $10 \mathrm{mM}$ EGTA, followed by an overnight incubation at $37^{\circ} \mathrm{C}$. Positive control was phage $\mathrm{P} 22$, and negative control was sterile water; both controls were used in all the steps. Transduction frequencies were calculated as the ratio of the number of transductants/PFU added for transduction $[65,83]$. Experiments were conducted in two independent replicates; if transduction was observed in at least one of the replicates, this phage was classified as a transducing phage.

\section{Availability of supporting data}

The sequence data supporting the results of this article are available on GenBank under the following accession numbers [GenBank: KC139511 to GenBank: KC139680].

\section{Additional files}

\begin{abstract}
Additional file 1: List of previously sequenced Salmonella phages used for comparative analysis. PDF file containing the list of previously sequenced phages used for comparative analysis.
\end{abstract}

Additional file 2: Summary of annotations in the different phages. PDF file containing a summary of functionally annotated ORFs in the phages sequenced here.

Additional file 3: Comparison, using the BLAST algorithm, of novel phages in cluster 1. PDF file containing the comparison of phages in cluster 1. Phages and farm origins are in the right side of the figure. Orange arrows indicate open reading frames (ORFs); grey shaded regions indicate regions with homology.

Additional file 4: Comparison, using the BLAST algorithm, of FSL SP-088 and Enterobacter phage Enc34. PDF file containing the comparison using the BLAST algorithm of FSL SP-088 and Enterobacter phage Enc34. Orange arrows indicate ORFs, and regions of homology are shaded in grey.

Additional file 5: Comparison, using the BLAST algorithm, of phages in cluster 5. PDF file containing the comparison of phages in cluster 5, representing four phages sequenced in this study (FSL SP-031, FSL SP-038, FSL SP-049 and FSL SP-101) and three phages previously sequenced in South Korea and U.K. Phages and country of origin are indicated on the right, purple arrows indicate ORFs, and grey shaded regions are regions of homology. On the left side is the tree generated with the Mauve algorithm indicating the overall similarity of the phages, this tree identified two branches named as 1 and 2 .

Additional file 6: Pairwise comparison of the amino acid identities on tailspikes and fibers. PDF file containing the percentage of amino acid identity of tailspikes and fibers in phage clusters.

Additional file 7: Host range of sequenced phage clusters. PDF file containing the host range of sequenced phages in clusters.

Additional file 8: Sequence data. Text file containing gbk files of all 22 sequenced phage genomes.

Competing interests

The authors declare that they have no competing interests.

\section{Authors' contributions}

AIMS, CA, MW: designed experiments; AIMS: conducted experiments; HB: participated in the sequences assembly; AIMS, RHO, HB, KV, MW: participated in genome analysis, and data interpretation; AIMS, MW: wrote the manuscript. All authors read and approved the final manuscript.

\section{Acknowledgements}

We thank the Felix d'Hérelle Reference Center (Université Laval, Quebec, Canada) for providing FelixO1. We also thank Dr. Haley Oliver for her help organizing the phage genome sequencing with Cornell University Life Sciences Core Laboratories Center, and we thank Thomas Denes for kindly reading the paper. This work was supported through a USDA-NIFA Special Research Grant to MW (2009-34459-19750). The funding source had no role in the study design; in the collection, analysis, and interpretation of data; in the writing of the report; and in the decision to submit the paper for publication.

\section{Author details}

${ }^{1}$ Department of Food Science, Cornell University, Ithaca 14853, New York. ${ }^{2}$ Department of Population Medicine and Diagnostic Sciences, College of Veterinary Medicine, Cornell University, Ithaca, New York. 
Received: 18 March 2013 Accepted: 11 July 2013

Published: 17 July 2013

\section{References}

1. Majowicz SE, Musto J, Scallan E, Angulo FJ, Kirk M, O'Brien SJ, Jones TF, Fazil A, Hoekstra RM: The global burden of nontyphoidal Salmonella gastroenteritis. Clin Infect Dis 2010, 50:882-889.

2. Scallan E, Hoekstra RM, Angulo FJ, Tauxe RV, Widdowson MA, Roy SL, Jones $J \mathrm{~L}$, Griffin PM: Foodborne illness acquired in the United States-major pathogens. Emerg Infect Dis 2011, 17:7-15.

3. Hoelzer K, Moreno Switt Al, Wiedmann M: Animal contact as a source of human non-typhoidal salmonellosis. Vet Res 2011, 42:34

4. Cummings KJ, Warnick LD, Elton M, Gröhn YT, McDonough PL, Siler JD: The effect of clinical outbreaks of salmonellosis on the prevalence of fecal Salmonella shedding among dairy cattle in New York. Foodborne Pathog Dis 2010, 7:815-823.

5. Van Kessel JA, Karns JS, Lombard JE, Kopral CA: Prevalence of Salmonella enterica, Listeria monocytogenes, and Escherichia coli virulence factors in bulk tank milk and in-line filters from U.S. dairies. J Food Prot 2011, 74:759-768.

6. $[C D C]$ Center for Disease Control and Prevention: Salmonella: annual summary (2009). Atlanta, GA: CDC, Division of Bacterial and Mycotic Diseases; 2007. http://www.cdc.gov/ncezid/dfwed/PDFs/ SalmonellaAnnualSummaryTables2009.pdf.

7. Moreno Switt Al, den Bakker HC, Vongkamjan K, Hoelzer K, Warnick LD Cummings K, Wiedmann M: Salmonella bacteriophage diversity reflects host diversity on dairy farms. Food Microbiol 2013, 36:275-285.

8. Kropinski AM, Sulakvelidze A, Konczy P, Poppe C: Salmonella phages and prophages-genomics and practical aspects. Methods Mol Biol 2007, 394:133-75

9. Suttle CA: Marine viruses-major players in the global ecosystem. Nat Rev Microbiol 2007, 5:801-12

10. Casjens SR, Gilcrease EB, Winn-stapley DA, Schicklmaier P, Schmieger H, Pedulla ML, Ford ME, Houtz JM, Hatfull GF, Hendrix RW: The generalized transducing Salmonella bacteriophage ES18: complete genome sequence and DNA packaging strategy. J Bacteriol 2005, 187:1091-1104.

11. Weinbauer MG, Rassoulzadegan F: Are viruses driving microbial diversification and diversity? Environ Microbiol 2004, 6:1-11.

12. Hooton SP, Timms AR, Rowsell J, Wilson R, Connerton IF: Salmonella Typhimurium-specific bacteriophage $\Phi S H 19$ and the origins of species specificity in the Vi01-like phage family. Virol J 2011, 8:498.

13. Price-Carter M, Roy-Chowdhury P, Pope CE, Paine S, de Lisle GW, Collins DM, Nicol C, Carter PE: The evolution and distribution of phage ST160 within Salmonella enterica serotype Typhimurium. Epidemiol Infect 2011, 139:1262-71.

14. Santos SB, Kropinski AM, Ceyssens PJ, Ackermann HW, Villegas A, Lavigne R, Krylov VN, Carvalho CM, Ferreira EC, Azeredo J: Genomic and proteomic characterization of the broad host range Salmonella phage PVP-SE1 - The creation of a new phage genus. J Virol 2011, 85:11265-73.

15. Lee JH, Shin H, Ryu S: Complete genome sequence of Salmonella enterica serovar Typhimurium bacteriophage SPN3UB. J Virol 2012, 86:3404-5.

16. Shin H, Lee JH, Lim JA, Kim H, Ryu S: Complete genome sequence of Salmonella enterica serovar Typhimurium bacteriophage SPN1S. J Virol 2012, 86:1284-5.

17. Jacobsen A, Hendriksen RS, Aaresturp FM, Ussery DW, Friis C: The Salmonella enterica pan-genome. Microb Ecol 2011, 62:487-504.

18. Den Bakker HC, Moreno Switt Al, Govoni G, Cummings CA, Ranieri ML, Degoricija L, Hoelzer K, Rodriguez-Rivera LD, Brown S, Bolchacova E, Furtado MR, Wiedmann M: Genome sequencing reveals diversification of virulence factor content and possible host adaptation in distinct subpopulations of Salmonella enterica. BMC Genomics 2011, 12:425.

19. Fricke WF, Mammel MK, McDermott PF, Tartera C, White DG, Leclerc JE, Ravel J, Cebula TA: Comparative genomics of 28 Salmonella enterica isolates: evidence for CRISPR-mediated adaptive sublineage evolution. J Bacteriol 2011, 193:3556-68

20. Lee JH, Shin H, Kim H, Ryu S: Complete genome sequence of Salmonella bacteriophage SPN3US. J Virol 2011, 85:13470-1.

21. Villegas A, She YM, Kropinski AM, Lingohr EJ, Mazzocco A, Ojha S, Waddell TE, Ackermann HW, Moyles DM, Ahmed R, Johnson RP: The genome and proteome of a virulent Escherichia coli 0157:H7 bacteriophage closely resembling Salmonella phage Felix O1. Virol J 2009, 6:41.
22. Pope WH, Jacobs-Sera D, Russell DA, et al: Expanding the diversity of mycobacteriophages: insights into genome architecture and evolution. PLoS One 2011, 6:e16329.

23. Hatfull GF, Jacobs-Sera D, Lawrence JG, Pope WH, Russell DA, Ko CC, Weber RJ, Patel MC, Germane KL, Edgar RH, Hoyte NN, Bowman CA, Tantoco AT, Paladin EC, Myers MS, Smith AL, Grace MS, Pham TT, O'Brien MB, Vogelsberger AM, Hryckowian AJ, Wynalek JL, Donis-Keller H, Bogel MW, Peebles CL, Cresawn SG, Hendrix RW: Comparative genomic analysis of 60 Mycobacteriophage genomes: genome clustering, gene acquisition, and gene size. J Mol Biol 2010, 397:119-43.

24. Cresawn SG, Bogel M, Day N, Jacobs-Sera D, Hendrix RW, Hatfull GF: Phamerator: a bioinformatic tool for comparative bacteriophage genomics. BMC Bioinformatics 2011, 12:395.

25. International commitee on virus taxonomy. Virus Taxonomy: Virus Taxonomy: 2012 Release (current). http://ictvonline.org/virusTaxonomy.asp? version $=2012 \& b h c p=1$

26. Bullas $L R$, Mostaghimi AR, Arensdorf JJ, Rajadas PT, Zuccarelli AJ: Salmonella phage PSP3, another member of the P2-like phage group. Virology 1992 188:414

27. Hoelzer K, Cummings KJ, Wright EM, Rodriguez-Rivera LD, Roof SE, Moreno Switt Al, Dumas N, Root T, Schoonmaker-Bopp DJ, Grohn YT, Siler JD, Warnick LD, Hancock DD, Davis MA, Wiedmann M: Salmonella Cerro isolated over the past twenty years from various sources in the US represent a single predominant pulsed-field gel electrophoresis type. Vet Microbiol 2011, 150:389-93.

28. Wang YC, Chang YC, Chuang HL, Chiu CC, Yeh KS, Chang CC, Hsuan SL, Lin WH, Chen TH: Transmission of Salmonella between swine farms by the housefly (Musca domestica). J Food Prot 2011, 74:1012-6.

29. Umali DV, Lapuz RR, Suzuki T, Shirota K, Katoh H: Transmission and shedding patterns of Salmonella in naturally infected captive wild roof rats (Rattus rattus) from a Salmonella-contaminated layer farm. Avian Dis 2012, 56:288-94.

30. Scholl D, Rogers S, Adhya S, Merril CR: Bacteriophage K1-5 encodes two different tail fiber proteins, allowing it to infect and replicate on both $\mathrm{K} 1$ and K5 strains of Escherichia coli. J Virol 2001, 75:2509-15.

31. Hatfull GF: Complete genome sequences of 138 mycobacteriophages. J Virol 2012, 86:2382-4.

32. Shin H, Lee JH, Kim H, Choi Y, Heu S, Ryu S: Receptor diversity and host interaction of bacteriophages infecting Salmonella enterica serovar Typhimurium. PLoS One 2012, 7:e43392.

33. Ladero V, García P, Alonso JC, Suárez JE: Interaction of the Cro repressor with the lysis/lysogeny switch of the Lactobacillus casei temperate bacteriophage A2. J Gen Virol 2002, 83:2891-5.

34. Das M, Ganguly T, Chattoraj P, Chanda PK, Bandhu A, Lee CY, Sau S: Purification and characterization of repressor of temperate $S$. aureus phage phi11.J Biochem Mol Biol 2007, 40:740-8.

35. Kazaks A, Dislers A, Lipowsky G, Nikolajeva V, Tars K: Complete Genome Sequence of the Enterobacter cancerogenus Bacteriophage Enc34. J Virol 2012, 86:11403-4.

36. Anany H, Lingohr EJ, Villegas A, Ackermann HW, She YM, Griffiths MW, Kropinski AM: A Shigella boydii bacteriophage which resembles Salmonella phage Vil. Virol J 2011, 8:242.

37. Adriaenssens EM, Ackermann HW, Anany H, Blasdel B, Connerton IF, Goulding D, Griffiths MW, Hooton SP, Kutter EM, Kropinski AM, Lee JH, Maes M, Pickard D, Ryu S, Sepehrizadeh Z, Shahrbabak SS, Toribio AL, Lavigne R: A suggested new bacteriophage genus: "Viunalikevirus". Arch Virol 2012, 157:2035-2046.

38. Pickard D, Toribio AL, Petty NK, van Tonder A, Yu L, Goulding D, Barrell B, Rance R, Harris D, Wetter M, Wain J, Choudhary J, Thomson N, Dougan G: A conserved acetyl esterase domain targets diverse bacteriophages to the Vi capsular receptor of Salmonella enterica serovar Typhi. J Bacteriol 2010, 192:5746-54

39. Park M, Lee JH, Shin H, Kim M, Choi J, Kang DH, Heu S, Ryu S: Characterization and comparative genomic analysis of a novel bacteriophage SFP10 simultaneously inhibiting both Salmonella and Escherichia coli O157:H7. Appl Environ Microbiol 2012, 78:58-69.

40. Whichard JM, Weigt LA, Borris DJ, Li LL, Zhang Q, Kapur V, Pierson FW, Lingohr EJ, She YM, Kropinski AM, Sriranganathan N: Complete genomic sequence of bacteriophage Felix 01. Viruses 2010, 2:710-30.

41. Lehman SM, Kropinski AM, Castle AJ, Svircev AM: Complete genome of the broad-host-range Erwinia amylovora phage phiEa21-4 and its 
relationship to Salmonella phage Felix 01. Appl Environ Microbiol 2009, 75:2139-47.

42. Edgell DR, Gibb EA, Belfort M: Mobile DNA elements in T4 and related phages. Virol J 2010, 7:290.

43. De Lappe N, Doran G, O'Connor J, O'Hare C, Cormican M: Characterization of bacteriophages used in the Salmonella enterica serovar Enteritidis phage-typing scheme. J Med Microbiol 2009, 58:86-93.

44. Gorbalenya AE, Koonin EV, Donchenko AP, Blinov VM: Two related superfamilies of putative helicases involved in replication, recombination, repair and expression of DNA and RNA genomes. Nucleic Acids Res 1989, 17:4713-30.

45. Thurber RV: Current insights into phage biodiversity and biogeography. Curr Opin Microbiol 2009, 12:582-7.

46. Ceyssens PJ, Miroshnikov K, Mattheus W, Krylov V, Robben J, Noben JP, Vanderschraeghe S, Sykilinda N, Kropinski AM, Volckaert G, Mesyanzhinov V, Lavigne R: Comparative analysis of the widespread and conserved PB1-like viruses infecting Pseudomonas aeruginosa. Environ Microbiol 2009, 11:2874-83

47. Ceyssens PJ, Glonti T, Kropinski NM, Lavigne R, Chanishvili N, Kulakov L, Lashkhi N, Tediashvili M, Merabishvili M: Phenotypic and genotypic variations within a single bacteriophage species. Virol J 2011, 23:134

48. The Mycobacterium database: Phage Isolation Locations by GPS Coordinates. http://phagesdb.org/GPSmap/.

49. The Mycobacterium database: Details for Subcluster A2 Phages. http:// phagesdb.org/subclusters/A2/.

50. Casjens SR, Thuman-Commike PA: Evolution of mosaically related tailed bacteriophage genomes seen through the lens of phage P22 virion assembly. Virology 2011, 411:393-415.

51. Mmolawa PT, Schmieger H, Heuzenroeder MW: Bacteriophage ST64B, a genetic mosaic of genes from diverse sources isolated from Salmonella enterica serovar Typhimurium DT 64. J Bacteriol 2003, 185:6481-5.

52. Loś JM, Loś M, Węgrzyn G: Bacteriophages carrying shiga toxin genes: genomic variations, detection and potential treatment of pathogenic bacteria. Future Microbiol 2011, 6:909-24.

53. Brüssow H, Canchaya C, Hardt WD: Phages and the evolution of bacterial pathogens: from genomic rearrangements to lysogenic conversion. Microbiol Mol Biol Rev 2004, 68:560-602.

54. Stanley $\mathrm{TL}$, Ellermeier $C D$, Slauch JM: Tissue-specific gene expression identifies a gene in the lysogenic phage Gifsy-1 that affects Salmonella enterica serovar Typhimurium survival in Peyer's patches. J Bacteriol 2000, 182:4406-13.

55. Mirold S, Rabsch W, Tschäpe H, Hardt WD: Transfer of the Salmonella type III effector sopE between unrelated phage families. J Mol Biol 2001 312:7-16.

56. Mirold S, Rabsch W, Rohde M, Stender S, Tschäpe H, Rüssmann H, Igwe E, Hardt WD: Isolation of a temperate bacteriophage encoding the type III effector protein SopE from an epidemic Salmonella Typhimurium strain Proc Natl Acad Sci USA 1999, 96:9845-50.

57. Cardinale CJ, Washburn RS, Tadigotla VR, Brown LM, Gottesman ME, Nudler E: Termination factor Rho and its cofactors NusA and NusG silence foreign DNA in E. coli. Science 2008, 320:935-8.

58. Kohn H, Widger W: The molecular basis for the mode of action of bicyclomycin. Curr Drug Targets Infect Disord 2005, 5:273-95.

59. Laskaris P, Sekine T, Wellington EM: Diversity analysis of Streptomycetes and associated phosphotranspherase genes in soil. PLoS One 2012, 7:e35756.

60. Chiang SK, Lou YC, Chen C: NMR solution structure of KP-TerB, a telluriteresistance protein from Klebsiella pneumoniae. Protein Sci 2008, 17:785-9.

61. Johnson TJ, Wannemeuhler YM, Scaccianoce JA, Johnson SJ, Nolan LK: Complete DNA sequence, comparative genomics, and prevalence of an IncHI2 plasmid occurring among extraintestinal pathogenic Escherichia coli isolates. Antimicrob Agents Chemother 2006, 50:3929-33.

62. Hoiczyk E, Roggenkamp A, Reichenbecher M, Lupas A, Heesemann J: Structure and sequence analysis of Yersinia YadA and Moraxella UspAs reveal a novel class of adhesins. EMBO J 2000, 19:5989-99.

63. Molina-Quiroz RC, Muñoz-Villagrán CM, de la Torre E, Tantaleán JC, Vásquez CC. Pérez-Donoso JM: Enhancing the antibiotic antibacterial effect by sub lethal tellurite concentrations: tellurite and cefotaxime act synergistically in Escherichia coli. PLoS One 2012, 7:e35452.

64. Schicklmaier $\mathrm{P}, \mathrm{Schmieger} \mathrm{H}$ : Frequency of generalized transducing phages in natural isolates of the Salmonella Typhimurium complex. Appl Environ Microbiol 1995, 61:1637-40.
65. Sternberg NL, Maurer R: Bacteriophage-mediated generalized transduction in Escherichia coli and Salmonella Typhimurium. Methods Enzymol 1991, 204:18-43

66. Waddell TE, Franklin K, Mazzocco A, Kropinski AM, Johnson RP: Generalized transduction by lytic bacteriophages. Methods Mol Biol 2009, 501:293-303.

67. Miller ES, Kutter E, Mosig G, Arisaka F, Kunisawa T, Rüger W: Bacteriophage T4 genome. Microbiol Mol Biol Rev 2003, 67:86-156.

68. Guttman BS, Kutter EM: Bacteriophage genetics, in modern microbial genetics. 2nd edition. New York, USA: Edited by U. N. Streips and R. E. Yasbin. John Wiley \& Sons, Inc.; 2002.

69. Casjens SR, Molineux IJ: Short noncontractile tail machines: adsorption and DNA delivery by podoviruses. Adv Exp Med Biol 2012, 726:143-79.

70. Davidson AR, Cardarelli L, Pell LG, Radford DR, Maxwell KL: Long noncontractile tail machines of bacteriophages. Adv Exp Med Biol 2012, 726:115-42.

71. Welkos S, Schreiber M, Baer H: Identification of Salmonella with the O-1 bacteriophage. Appl Microbiol 1974, 28:618-22.

72. Sambrook J, Russell DW: Molecular cloning. 3rd edition. New York: Cold Spring Harbor Laboratory Press; 2001.

73. Zerbino DR, Birney E: Velvet: algorithms for de novo short read assembly using de Bruijn graphs. Genome Res 2008, 18:821-9.

74. Aziz RK, Bartels D, Best AA, DeJongh M, Disz T, Edwards RA, Formsma K, Gerdes S, Glass EM, Kubal M, Meyer F, Olsen GJ, Olson R, Osterman AL, Overbeek RA, McNeil LK, Paarmann D, Paczian T, Parrello B, Pusch GD, Reich C, Stevens R, Vassieva O, Vonstein V, Wilke A, Zagnitko O: The RAST Server: rapid annotations using subsystems technology. BMC Genomics 2008, 9:75.

75. NCBI Prokaryotic Genomes Automatic Annotation Pipeline (PGAAP). http:// www.ncbi.nlm.nih.gov/genomes/static/Pipeline.html.

76. Wiki food safety. https://confluence.cornell.edu/display/FOODSAFETY/ Cornell+Food+Safety+Laboratory+Microbial+Genome+Data.

77. Li L, Stoeckert CJ, Roos DS: OrthoMCL: identification of ortholog groups for eukaryotic genomes. Genome Res 2003, 13:2178-89.

78. Huson DH, Bryant D: Application of phylogenetic networks in evolutionary studies. Mol Biol Evol 2006, 23:254-67.

79. Krumsiek J, Arnold R, Rattei T: Gepard: a rapid and sensitive tool for creating dotplots on genome scale. Bioinformatics 2007, 23:1026-8.

80. Darling ACE, Mau B, Blattner FR, Perna NT: Mauve: multiple alignment of conserved genomic sequence with rearrangements. Genome Res 2004, 14:1394-403.

81. Sullivan MJ, Petty NK, Beatson SA: Easyfig: a genome comparison visualizer. Bioinformatics 2011, 27:1009-10.

82. Camacho C, Coulouris G, Avagyan V, Ma N, Papadopoulos J, Bealer K, Madden TL: BLAST+: architecture and applications. BMC Bioinformatics 2009, 10:421.

83. Petty NK, Toribio AL, Goulding D, Foulds I, Thomson N, Dougan G, Salmond GP: A generalized transducing phage for the murine pathogen Citrobacter rodentium. Microbiol 2007, 153:2984-8.

doi:10.1186/1471-2164-14-481

Cite this article as: Moreno Switt et al:: Genomic characterization provides new insight into Salmonella phage diversity. BMC Genomics $201314: 481$

\section{Submit your next manuscript to BioMed Central and take full advantage of:}

- Convenient online submission

- Thorough peer review

- No space constraints or color figure charges

- Immediate publication on acceptance

- Inclusion in PubMed, CAS, Scopus and Google Scholar

- Research which is freely available for redistribution 